\title{
Modelagem e Otimização do Leito de Fusão do Alto-forno na Aperam
}

\author{
Alcy Dias Rodrigues* Tales Wallace Souza** \\ Rodney Resende Saldanha ${ }^{* * *}$ Vinicius Mariano Gonçalves ${ }^{* * *}$ \\ * Programa de Pós Graduação em Engenharia Elétrica, Universidade \\ Federal de Minas Gerais, MG (e-mail: alcy.rodrigues@aperam.com). \\ ** Gerência Executiva de Automação e Infraestrutura, Aperam South \\ America, $M G$ (e-mail: tales.souza@aperam.com) \\ *** Departamento de Engenharia Elétrica, Universidade Federal de \\ Minas Gerais, MG (e-mail: rodney@cpdee.ufmg.br, \\ mariano@cpdee.ufmg.br)
}

\begin{abstract}
This paper presents the proposal of an optimization model that aims to minimize the slag volume of Aperam blast furnaces. Currently, the furnace team uses a mathematical model to calculate the mass balance, called the Burden Calculation, which does not optimize any of their variables. Still, a manual adjustment of the parameters of the model by the operational team is necessary, following limits established by the metallurgists. Through the identification of the nonlinear functions of the Burden Calculation, a linear regression was applied to model a Mixed Integer Linear Programming problem. This model is solved using a technique similar to Sequential Linear Programming. The results presented demonstrate the efficiency of the model, which not only eliminated the necessity of manual adjustment but also presented lower slag volume for all the Burden Calculation and also obtained a reduction in the consumption of raw materials.

Resumo: Este artigo apresenta a proposta de um modelo de otimização que objetiva minimizar o volume de escória dos Alto-fornos da Aperam. Atualmente, a equipe do forno utiliza um modelo matemático para cálculo do balanço de massa, denominado Leito de Fusão, que não realiza nenhum tipo de otimização nas variáveis do modelo. Ainda, é necessário um ajuste manual nos parâmetros do modelo pela equipe operacional, seguindo limites estabelecidos pelos metalurgistas. Através da identificação das funções não lineares do Leito de Fusão, foi aplicado uma regressão linear para modelagem de um problema de Programação Linear Inteira Mista. Esse modelo é resolvido utilizando uma técnica similar à Programação Linear Sequencial. Os resultados apresentados demostram a eficiência do modelo, que não só eliminou a necessidade de ajuste manual, mas também apresentou menor volume de escória para todos os Leitos de Fusão e obteve uma redução no consumo de matérias-primas.
\end{abstract}

Keywords: Optimization Model, Slag volume, Blast Furnace, Burden Calculation, Linear Regression, Mixed Integer Linear Programming, Sequential Linear Programming.

Palavras-chaves: Modelo de Otimização, Volume de Escória, Alto-forno, Leito de Fusão, Regressão Linear, Programação Linear Inteira Mista, Programação Linear Sequencial.

\section{INTRODUÇÃO}

Em uma usina siderúrgica integrada, o alto-forno é responsável pela produção de ferro-gusa. Para isso, utilizase do carvão vegetal ou coque como combustível, minério e fundentes como carga metálica. No processo do altoforno toda carga enfornada transforma-se em ferro-gusa e escória. O alto-forno funciona de forma contínua, ou seja, não deve ter sua produção paralisada, a não ser para realização de manutenções programadas em equipamentos considerados críticos para o seu funcionamento seguro.

Segundo Geerdes et al. (2015), para que a operação do altoforno seja efetuada com boa produtividade, imprescindível segurança dos funcionários e equipamentos, estabilidade operacional, menor consumo de combustível, requisitos de qualidade especificados pelo cliente e a não ocorrência de eventos danosos ao meio-ambiente, além disso é de suma importância que se obtenha uma boa estabilidade operacional. Resultados operacionais excelentes em um alto-forno só poderão ser alcançados se três condições forem satisfeitas:

- Boa qualidade da carga e do carvão.

- Bom controle do fluxo de gás.

- Boa prática de escoamento de gusa.

Em pesquisa realizada, foi identificado diversas abordagens relacionadas à otimização da distribuição de carga, que está relacionado com o primeiro aspecto citado. Wu et al. (2018) apresenta uma abordagem de tomada de decisão para escolha da melhor distribuição de carga em um alto- 
forno, objetivando o menor consumo de energia. Yang et al. (2017) apresenta um modelo para controle de processo da distribuição de carga em alto-forno, objetivando otimização dinâmica da produção e mantendo a estabilidade operacional do alto-forno.

Em outro trabalho, Wu et al. (2010) apresenta um modelo matemático para otimização da distribuição de carga, objetivando a redução do custo do ferro-gusa. Zhang et al. (2018) apresenta a abordagem de utilização do método de otimização por enxame de partículas (PSO) para otimização da distribuição de carga, baseado na complexa relação entre a entrada e saída de carga.

Este trabalho está interessado em proporcionar uma melhoria no terceiro aspecto, isto é, melhorar as condições de escoamento do gusa. Para isso, um aspecto importante é o leito de fusão do forno, que é um balanço de massa que objetiva descobrir qual será a quantidade de materiais (carga metálica, fundentes e combustível) que serão enfornadas atendendo as especificações da Aciaria.

Atualmente esse procedimento de execução do balanço massa é realizado pelos operadores do forno utilizando um modelo matemático implementado no sistema de automação. Entretanto, o sistema implementado não contempla todas as restrições operacionais do forno, exigindo do operador ajustes manuais na resposta do sistema. Esses ajustes são complexos e demandam bastante tempo do operador.

Nesse sentido, este trabalho propõe uma mudança no modelo matemático implementado, objetivando não só contemplar as restrições que o sistema atual não contempla como também otimizar um importante aspecto operacional do forno: o volume de escória. Verifica-se que um menor volume de escória - dentro de uma faixa de segurança que não prejudique as condições de drenagem e esgotamento - fornece maior estabilidade operacional para o forno e também um aumento de produtividade conforme apresentado por Geerdes et al. (2015). Propõe-se então um modelo matemático que respeita as restrições operacionais do forno e visa minimizar o volume de escoria.

O modelo desenvolvido tem como base as equações do leito de fusão implementadas no sistema de automação. Essas equações complexas, não lineares, serão linearizadas para que seja possível escrever um modelo de programação linear inteira mista. Um procedimento similar à estratégia de programação linear sequencial será utilizada para garantir que a resposta do modelo linearizado não seja inválida em relação ao modelo original. Será feita uma discussão acerca dos aspectos técnicos do forno, tal qual como da solução desenvolvida. Mostraremos que, com a abordagem proposta, não só conseguimos automatizar o processo de realizar o cálculo leito de fusão - respeitando todas as restrições - mas como também fornecer condições operacionais mais favoráveis ao alto-forno.

\section{O ALTO-FORNO}

\subsection{Principio de funcionamento do Alto-forno}

O alto-forno é um reator metalúrgico empregado na produção de ferro-gusa, através da fusão redutora de minérios de ferro em presença de carvão vegetal ou coque e

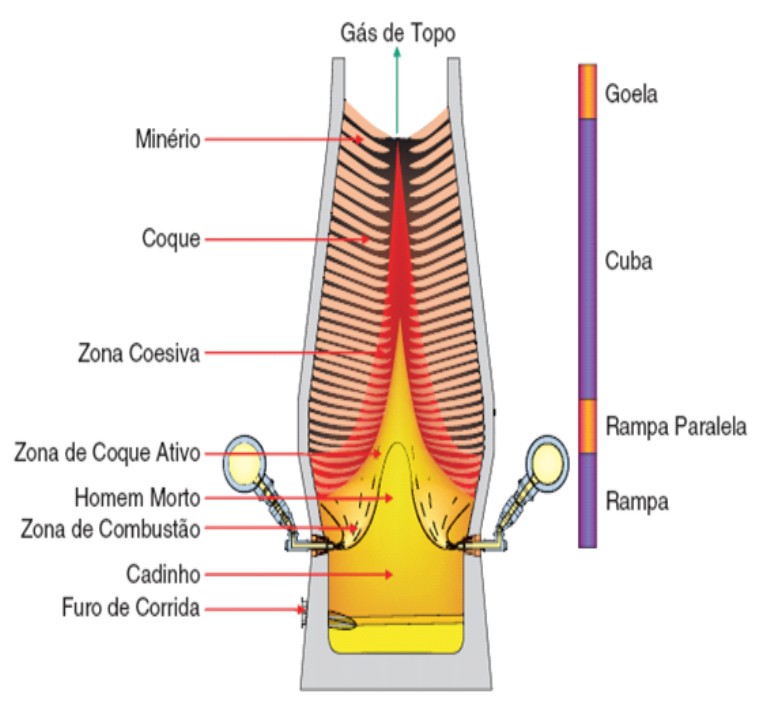

Figura 1. Alto-forno. Geerdes et al. (2015)

fundentes. Estes materiais são carregados pelo topo na temperatura ambiente e, na descida, são transformados pela ação dos gases ascendentes, provenientes da reação de combustão do carbono dos combustíveis com o oxigênio do ar de combustão aquecido (500 a $1200^{\circ} \mathrm{C}$ ou mais) soprado pelas ventaneiras, enriquecido ou não com oxigênio e/ou combustíveis auxiliares, obtendo-se escória e o ferro-gusa, depositados no cadinho e as poeiras e os gases no topo, conforme da Silveira Rizzo (2009). No interior de um altoforno em funcionamento existe, de cima para baixo:

- Camadas de minério e carvão.

- Uma região onde o minério e fundentes começam a amolecer.

- Uma região onde existem, da carga, apenas carvão sólido e líquidos, nas regiões de gotejamento ou carvão ativo.

- Um local onde o metal e a escória líquidos são acumulados nos vazios entre as partículas de carvão (cadinho).

Conforme Geerdes et al. (2015), o alto-forno é constituído por uma carcaça metálica revestida internamente por refratários apresentando as seguintes regiões, como apresentado na Figura 1:

- Goela.

- Cuba.

- Ventre.

- Rampa.

- Cadinho.

A força motriz do processo do alto-forno são os gases gerados pela combustão do carbono do redutor sólido na região inferior do forno através de sua reação com o oxigênio do ar quente injetado em alta velocidade pelas ventaneiras. Os gases gerados nesta região fluem para cima no alto-forno, arrastando materiais particulados da carga ferrífera, fundentes e combustível. Os gases reagem com a carga ferrífera que estão se deslocando em contracorrente promovendo progressivamente a redução dos óxidos de ferro, obtendo-se o ferro metálico e gerando uma quantidade adicional de gás. As reações químicas de combustão e de redução geram energia suficiente para fundir o 
ferro metálico e a escória gerada no processo. Para que estas reações ocorram de uma forma que seja atingido um baixo consumo de combustível, elevada produtividade e alta segurança e estabilidade operacional, é fundamental que a carga sólida seja homogênea química e granulometricamente e, além do mais, que apresente uma distribuição bem definida, para permitir a passagem dos gases de forma adequada, facilitando assim as trocas térmicas e químicas. Estes materiais, no estado líquido, escoam para o fundo do forno para uma região denominada de cadinho conforme apresentado por Geerdes et al. (2015).

Para obtenção de uma boa qualidade da carga e do carvão e atingir uma boa prática de escoamento de gusa, faz-se necessário estar atendo a todos requerimentos de carga. No processo de carregamento do alto-forno, o mesmo possuí um modelo matemático, denominado leito de fusão (balanço de massa completo) que realiza o cálculo para o carregamento das cargas metálicas no interior do mesmo. Estes cálculos são realizados pelos operadores, fazendo-se necessário um recálculo a cada alteração no resultado da análise química.

A validação do resultado ocorre quando as principais variáveis (volume de escória, $\mathrm{MgO}$, basicidade) encontramse dentro dos limites definidos a priori pelos metalurgistas e não apresente valor de peso negativo para os fundentes. Caso contrário, faz-se necessário vários recálculos até que os resultados estejam dentro destes limites.

\subsection{Cálculo do Leito de Fusão}

Atualmente o cálculo do leito de fusão do alto-forno é realizado no sistema de nível 2, conforme pirâmide da Automação Industrial (modelo matemático), cálculo este realizado para atender as especificação da qualidade do ferro-gusa e escória. O alto-forno é carregado com minério e carvão vegetal. A aciaria, área responsável pelo recebimento do ferro-gusa da Redução para produção do aço, requer uma qualidade definida para o ferro-gusa e a escória que tem que ser escolhida pelas propriedades otimizadas com relação à fluidez. Sendo assim, o operador do alto-forno tem que fazer cálculos para escolher a carga do alto-forno.

O modelo matemático do leito de fusão está implementado no sistema de Nível 2 do forno. Os sistemas de nível 2 são sistemas que contém as informações provenientes do campo, onde são armazenadas, objetivando atender as necessidades da equipe de operação e controle de processo, fornecendo informações do processo produtivo para auxiliar nas tomadas de decisões. Este sistema possui uma arquitetura cliente/servidor em que o cliente é apenas uma interface de integração com o usuário e o servidor contém toda a lógica de regra do negócio e modelagem matemática.

\section{METODOLOGIA}

\subsection{Modelagem geral e estratégia de otimização}

O problema do leito de fusão será modelado como um problema de otimização. As variáveis de decisão do nosso problema são:
- Volume de escória: v

- Basicidade: b

- Óxido de Magnésio (MgO): m

Estas variáveis são referência para obtenção de uma escória que proporcione maior estabilidade operacional ao reator, pois as mesmas estão diretamente relacionadas com viscosidade da escória. A viscosidade é uma propriedade física, que caracteriza a resistência de um fluido ao escoamento a uma dada temperatura, ou seja, com baixa viscosidade temos maior fluidez.

Há no problema três cargas metálicas, HGVB, HGSB, PACB e quatro fundentes, MMN2, QZ01, KCA2, DOLB. A quantidade de todos esses materiais estão em função das três variáveis de decisão e dos parâmetros do problema e essa relação é obtida de cálculos metalúrgicos complexos feitos por um especialista. Portanto, ao decidir as três variáveis de decisão, o modelo nos fornece quanto desses materiais deve ser utilizado no forno.

Nossa função objetivo é minimizar $v$, o volume de escória, por se tratar de uma das principais características da escória. O ideal é trabalhar com o menor volume de escória, desde que não prejudique as condições de drenagem e esgotamento do alto-forno. Ainda, é necessário considerar algumas restrições para a quantidade de fundentes e carga metálica. São elas: (i) a quantidade não pode ser negativa e (ii) se o material for utilizado, no mínimo $80 \mathrm{~kg}$ devem ser utilizados devido à limitação de precisão da balança. Isso é codificado matematicamente exigindo que a variável esteja no conjunto $\mathbb{R}$. Assim, temos o seguinte modelo conforme Equação 1.

$$
\begin{aligned}
& \min _{v, b, m, Z} v \text { sujeito às restrições } \\
& 80 Z_{H G V B} \leq \operatorname{HGVB}(v, b, m) \leq M Z_{H G V B} \\
& 80 Z_{H G S B} \leq \operatorname{HGSB}(v, b, m) \leq M Z_{H G S B} \\
& 80 Z_{P A C B} \leq \operatorname{PACB}(v, b, m) \leq M Z_{P A C B} \\
& 80 Z_{M M N 2} \leq \operatorname{MMN} 2(v, b, m) \leq M Z_{M M N 2} \\
& 80 Z_{Q Z O 1} \leq \mathrm{QZO} 1(v, b, m) \leq M Z_{Q Z O 1} \\
& 80 Z_{K C A 2} \leq \operatorname{KCA} 2(v, b, m) \leq M Z_{K C A 2} \\
& 80 Z_{D O L B} \leq \operatorname{DOLB}(v, b, m) \leq M Z_{D O L B} \\
& v_{\min } \leq v \leq v_{\max } \\
& b_{\min } \leq b \leq b_{\max } \\
& m_{\min } \leq m \leq m_{\max } \\
& v, b, m \in \mathbb{R}^{+} \\
& Z_{H G V B}, Z_{H G S B}, Z_{P A C B}, Z_{M M N 2} \\
& Z_{Q Z O 1}, Z_{K C A 2}, Z_{D O L B} \in\{0,1\}
\end{aligned}
$$

em que $M$ é um valor constante que será discutido em breve. Essas funções (HGVB, HGSB, PACB, MMN2, QZ01, KCA2 e DOLB) são, no geral, não lineares nas variáveis de decisão. Portanto, nossas restrições são não lineares e não convexas, uma vez que o conjunto $\{0\} \cup$ $[80, \infty)$ é não convexo. As variáveis $Z_{H G V B}, Z_{H G S B}$, $Z_{P A C B}, Z_{M M N 2}, Z_{Q Z O 1}, Z_{K C A 2}$ e $Z_{D O L B}$ são variáveis binárias utilizadas para limitar o valor das cargas devido à tara da balança, que é de $80 \mathrm{~kg}$. Ao assumir valor igual a 0 os pesos serão zerados. Ao assumir o valor 1, os pesos serão 
maior ou igual que $80 \mathrm{~kg}$ e menor ou igual ao valor de $M$. Uma vez que $M$ é escolhido sendo um valor muito grande (aqui assumimos $M=10000$ ), a restrição de ser menor ou igual ao valor de $M$ é inócua. Essa técnica é conhecida como técnica do grande $M$ (Griva et al. (2009)), e é clássica para implementar restrições de inserção em um conjunto não convexo.

Dessa forma, o problema é complexo e portanto iremos utilizar uma estratégia de linearização para resolvê-la, mais especificamente, uma estratégia baseada em programação linear sequencial (PLS) conforme apresentado em Nocedal and Wright (2006). Em cada iteração do método, o modelo não linear é linearizado em uma região (uma caixa no espaço das três variáveis) das variáveis de decisão (as três últimas restrições do modelo anterior) ao redor das variáveis ótimas $(v, b, m, Z)$ encontradas na iteração anterior. Essa região é sucessivamente menor a cada iteração. Essa técnica será apresentada com mais detalhes na próxima subseção.

\subsection{Técnica de otimização}

Discutiremos agora em mais detalhes a técnica de otimização descrita na subseção anterior. Ela segue o seguinte fluxo de passos:

(1) Estabelecemos limites iniciais para as variáveis de decisão de acordo com a experiência dos metalurgistas. Essa será nossa região inicial $\mathcal{R}$, uma caixa;

(2) Utilizando as funções não lineares das cargas metálicas e fundentes e a técnica de regressão linear, é gerado os modelos lineares aproximados para cada função (carga metálica e fundentes) na região $\mathcal{R}$;

(3) É montado um problema de programação linear inteira mista a partir do modelo 1. Esse modelo é resolvido utilizando o LPSOLVE (Berkelaar et al. (2004)). Obtemos a solução ótima atual $\left(v_{o t}, b_{o t}, m_{o t}, Z_{o t}\right)$;

(4) De posse da solução ótima, obtemos uma nova região $\mathcal{R}$, menor que a anterior, ao redor $\left(v_{o t}, b_{o t}, m_{o t}, Z_{o t}\right)$. Voltamos para o passo 2.

Para melhor entendimento da sequência de execução do algoritmo, apresenta-se na Figura 3 um fluxograma com a ordem de execução do modelo proposto para otimização do volume de escória.

No passo 2, é necessário linearizar o modelo 1 para obter um modelo de programação linear inteira mista (PLIM), conforme apresentado em Griva et al. (2009). Para isso, para cada função não linear (HGVB, HGSB, PACB, MMN2, QZ01, KCA2 e DOLB) e para cada região $\mathcal{R}$ em que será resolvido o problema de programação linear, é estimado um modelo linear da forma $y=\alpha_{v} v+\alpha_{b} b+$ $\alpha_{m} m+\beta$, nas três variáveis de decisão, que mais se ajusta ao modelo não linear. Para isso é utilizada a técnica de mínimos quadrados conforme apresentado por James et al. (2013). Portanto, após linearização, obtem-se o modelo conforme Equação 2, contendo as equações lineares:

$$
\begin{aligned}
& \min _{v, b, m, Z} v \text { sujeito às restrições } \\
& 80 Z_{H G V B} \leq \alpha_{v}^{H G V B} v+\alpha_{b}^{H G V B} b+\alpha_{m}^{H G V B} m+\beta^{H G V B} \\
& \alpha_{v}^{H G V B} v+\alpha_{b}^{H G V B} b+\alpha_{m}^{H G V B} m+\beta^{H G V B} \leq M Z_{H G V B} \\
& 80 Z_{H G S B} \leq \alpha_{v}^{H G S B} v+\alpha_{b}^{H G S B} b+\alpha_{m}^{H G S B} m+\beta^{H G S B} \\
& \alpha_{v}^{H G S B} v+\alpha_{b}^{H G S B} b+\alpha_{m}^{H G S B} m+\beta^{H G S B} \leq M Z_{H G S B} \\
& 80 Z_{P A C B} \leq \alpha_{v}^{P A C B} v+\alpha_{b}^{P A C B} b+\alpha_{m}^{P A C B} m+\beta^{P A C B} \\
& \alpha_{v}^{P A C B} v+\alpha_{b}^{P A C B} b+\alpha_{m}^{P A C B} m+\beta^{P A C B} \leq M Z_{P A C B} \\
& 80 Z_{M M N 2} \leq \alpha_{v}^{M M N 2} v+\alpha_{b}^{M M N 2} b+\alpha_{m}^{M M N 2} m+\beta^{M M N 2} \\
& \alpha_{v}^{M M N 2} v+\alpha_{b}^{M M N 2} b+\alpha_{m}^{M M N 2} m+\beta^{M M N 2} \leq M Z_{M M N 2} \\
& 80 Z_{Q Z O 1} \leq \alpha_{v}^{Q Z O 1} v+\alpha_{b}^{Q Z O 1} b+\alpha_{m}^{Q Z O 1} m+\beta^{Q Z O 1} \\
& \alpha_{v}^{Q Z O 1} v+\alpha_{b}^{Q Z O 1} b+\alpha_{m}^{Q Z O 1} m+\beta^{Q Z O 1} \leq M Z_{Q Z O 1} \\
& 80 Z_{K C A 2} \leq \alpha_{v}^{K C A 2} v+\alpha_{b}^{K C A 2} b+\alpha_{m}^{K C A 2} m+\beta^{K C A 2} \\
& \alpha_{v}^{K C A 2} v+\alpha_{b}^{K C A 2} b+\alpha_{m}^{K C A 2} m+\beta^{K C A 2} \leq M Z_{K C A 2} \\
& 80 Z_{D O L B} \leq \alpha_{v}^{D O L B} v+\alpha_{b}^{D O L B} b+\alpha_{m}^{D O L B} m+\beta^{D O L B} \\
& \alpha_{v}^{D O L B} v+\alpha_{b}^{D O L B} b+\alpha_{m}^{D O L B} m+\beta^{D O L B} \leq M Z_{D O L B} \\
& v_{\min } \leq v \leq v_{\max } \\
& b_{\min } \leq b \leq b_{\max } \\
& m_{\min } \leq m \leq m_{\max } \\
& v, b, m \in \mathbb{R}^{+} \\
& Z_{H G V B}, Z_{H G S B}, Z_{P A C B}, Z_{M M N 2} \\
& Z_{Q Z O 1}, Z_{K C A 2}, Z_{D O L B} \in\{0,1\}
\end{aligned}
$$

em que os $\alpha$ 's e $\beta$ 's, são os coeficientes de linearização para cada função na região $\mathcal{R}$ ao redor da solução ótima encontrada na iteração anterior. A cada iteração da técnica de PLS, os limites nas três últimas restrições são estreitados.

A Figura 2 ilustra o procedimento (com duas variáveis em vez de três). A cada passo, linearizamos as funções não lineares que geram o espaço factível original (curva verde claro) dentro do retângulo de limites, obtendo uma estimativa linearizada da região (polígono verde escuro). É então resolvido um problema de programação linear dentro do polígono, obtendo uma nova solução. Procedemos novamente utilizando agora uma região em torno do ponto obtido. O processo é repetido quatro vezes. Note que a cada vez que a iteração é repetida, a região linearizada (verde escuro) se aproxima mais da região real (verde claro) dentro da caixa de linearização.

\section{RESULTADOS E DISCUSSÃO}

Para obtenção dos resultados e comparações, foram coletados 100 leitos de fusão, representando diferentes contextos operacionais. Os erros no processo de linearização foram calculados conforme equação 3, apresentando pequenos valores, o que representa um bom ajuste dos dados.

$$
\epsilon_{i}=\frac{x_{i}-\bar{x}_{i}}{x_{i}^{\text {max }}-x_{i}^{\text {min }}}
$$

Nessa equação, $x_{i}$ representa a $i$-ésima amostra que foi gerada pelo modelo não linear que foram geradas dentro 


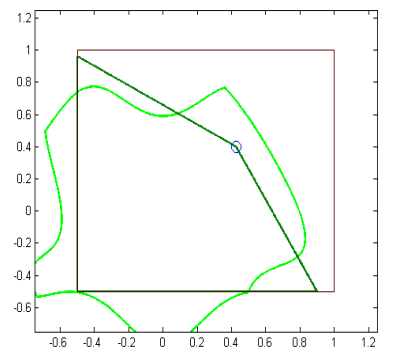

(a) Primeira iteração

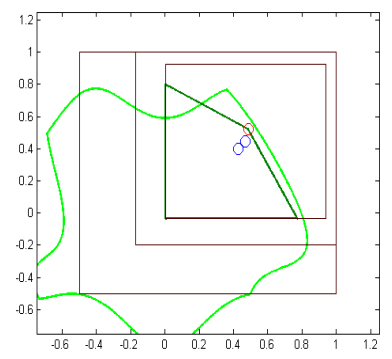

(c) Terceira iteração

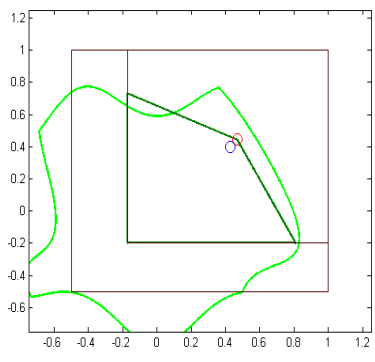

(b) Segunda iteração

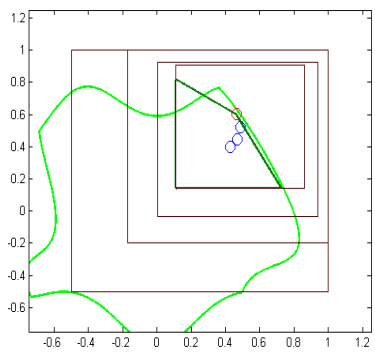

(d) Quarta iteração
Figura 2. Ilustração do algoritmo utilizado para fazer a otimização.

dos limites das variáveis de decisão $(\mathrm{v}, \mathrm{b}, \mathrm{m})$ e $x_{i}^{\max }, x_{i}^{\min }$ a variação máxima desses valores. Já a variável $\bar{x}_{i}$ corresponde ao $i$-ésimo valor estimado através do modelo linear obtido por mínimos quadrados.

Com base no resultado do erro para cada leito, foi efetuado uma média dos erros para cada função linearizada e em sequência foi extraído a média dos erros dos 100 leitos otimizados por iteração, conforme apresentado na Tabela 1, em que podemos observar a redução do erro de linearização à medida que aplicamos a programação linear sequencial, ou seja, a cada iteração reduzimos o erro máximo e erro médio.

Tabela 1.

\begin{tabular}{ccc} 
Iterações & Erro Máximo & Erro médio \\
\hline Primeira & $35,8 \%$ & $5,96 \%$ \\
Segunda & $39,2 \%$ & $4,08 \%$ \\
Terceira & $22,9 \%$ & $2,19 \%$ \\
Quarta & $7,8 \%$ & $0,7 \%$ \\
\hline
\end{tabular}

Conforme apresentado na Figura 4, que apresenta um gráfico box-plot da distribuição dos dados de erro, observase uma evolução na simetria dos valores, melhora na repartição entre os quartis e pouca influência de outliers a cada iteração, até alcançar o melhor ajuste aos dados.

$\mathrm{Na}$ Figura 5, observamos que para todos os 100 leitos otimizados, foram alcançados menores valores para volume de escória, que é a nossa função objetivo a ser minimizada.

Foi observado também que em função da redução do volume de escória, obtivemos uma redução na quantidade do Calcário e da Dolomita para cada cálculo do leito de fusão, conforme apresentado nas Figuras 6 e 7, o que representa uma redução de custo no processo de produção do ferro-gusa. Também podemos visualizar na Tabela 2, o percentual de redução das matérias primas, sendo: 4,8\%

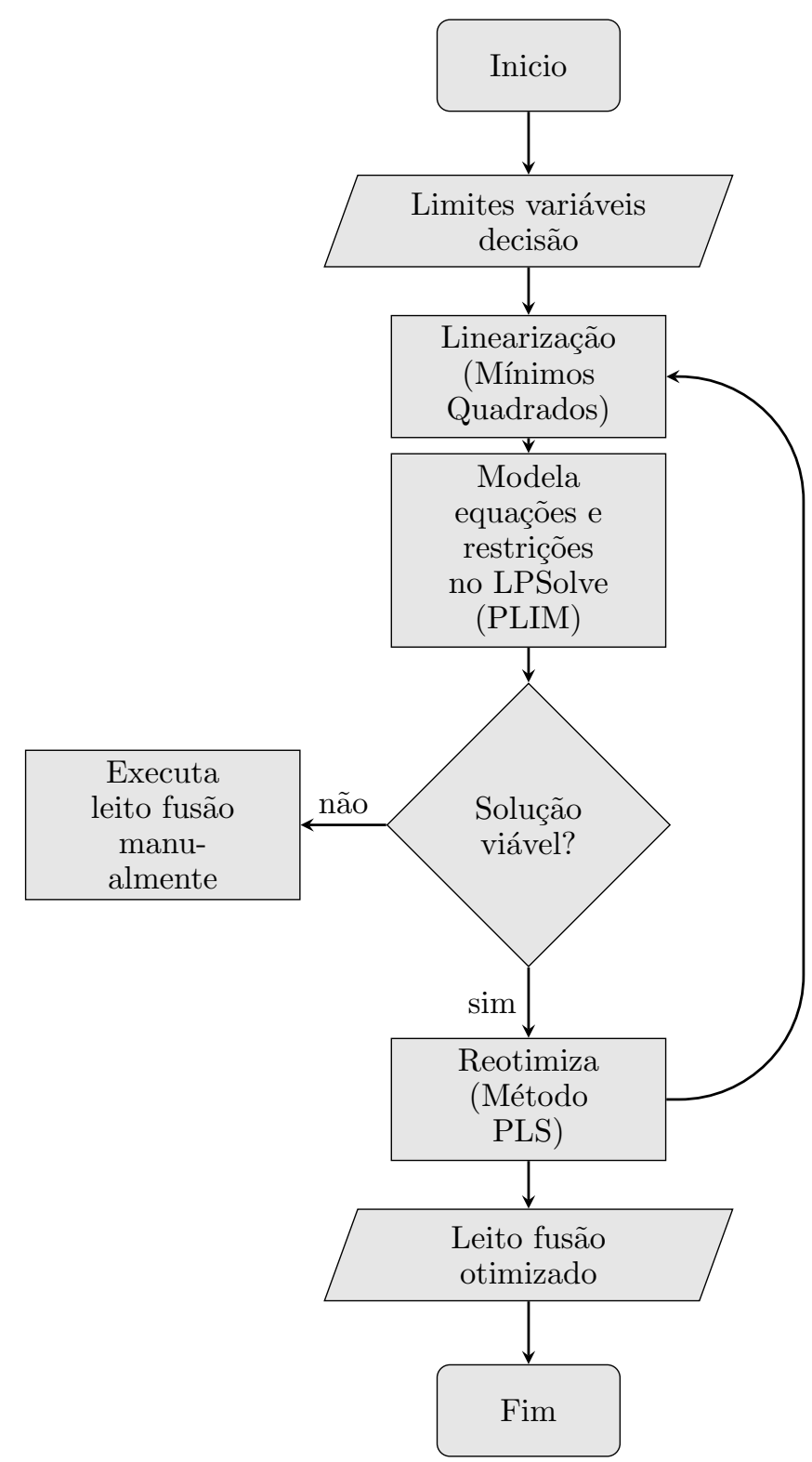

Figura 3. Sequência de execução do algoritmo

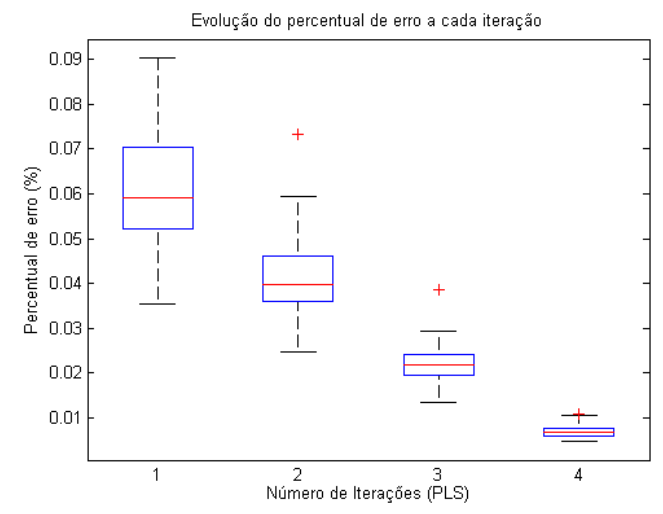

Figura 4. Evolução erro na PLS.

para Calcário (KCA2), 23\% para Dolomita (DOLB), e $4,7 \%$ no volume de escória, para os 100 leitos de fusão otimizados. 


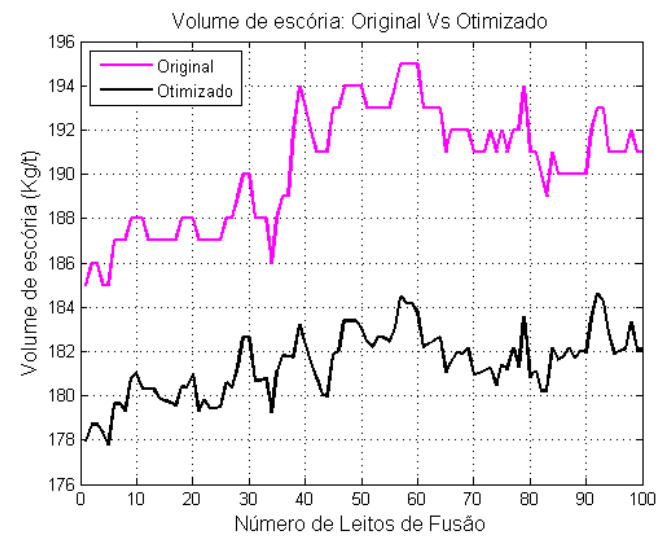

Figura 5. Resultado Volume Escória.

Tabela 2.

\begin{tabular}{cccc} 
Variável & Não linear & Otimizado & Redução \\
\hline HGVB (Minério) & 2497 & 2498 & $0 \%$ \\
HGSB (Minério) & 2497 & 2498 & $0 \%$ \\
PACB (Pelota) & 19979 & 19984 & $0 \%$ \\
MMN2 (Manganês) & 139 & 139 & $0 \%$ \\
QZO1 (Quartzo) & 0 & 0 & $0 \%$ \\
KCA2 (Calcário) & 603 & 574 & $4,8 \%$ \\
DOLB (Dolomita) & 996 & 764 & $23,0 \%$ \\
Al2O3(Alumina) & 12 & 12 & $0 \%$ \\
VolumeEscória & 190,35 & 181,4 & $4,7 \%$ \\
Basicidade & 0,76 & 0,7 & $7,8 \%$ \\
MgO (Óxido Magnésio) & 10 & 9 & $10,0 \%$ \\
\hline
\end{tabular}

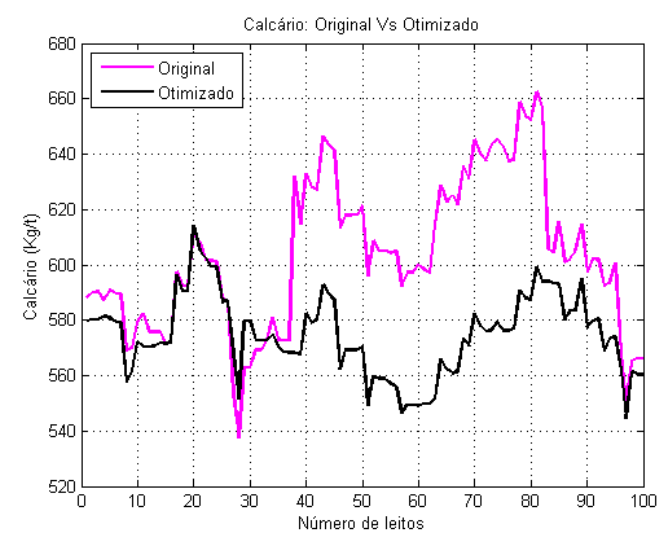

Figura 6. Redução de peso do Calcário.

\section{CONCLUSÃO}

O estudo do processo de carregamento do Alto-forno é complexo e envolve muitas variáveis, com várias restrições que devem ser respeitadas para garantia de uma boa estabilidade operacional e qualidade do produto final. O modelo proposto foi assertivo na utilização do método de PLIM e PLS para alcançarmos uma solução ótima para o problema.

Os resultados alcançados foram satisfatórios, em que alcançamos para todos os leitos de fusão uma significativa redução no volume de escória, que foi nossa função objetivo. Além disso, obtivemos também outros ganhos, como a redução na carga de fundentes por leito de fusão.

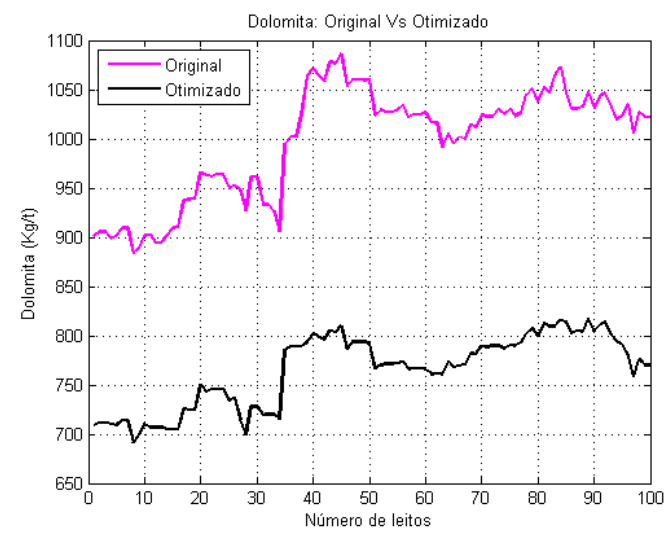

Figura 7. Redução de peso da Dolomita.

Todos os resultados já foram validados com a equipe do controle de processo da Aperam. O próximo passo é a conclusão da implantação do modelo no sistema de automação para que possa ser utilizado pelo operador.

\section{AGRADECIMENTOS}

Agradecemos à empresa Aperam Inox América do Sul, à Gerência Executiva de Automação e Infraestrutura, à Gerência do Controle de Processo da Redução e ao Programa de Pós-Graduação em Engenharia Elétrica da UFMG pelo apoio prestado em todas fases deste projeto.

\section{REFERÊNCIAS}

Berkelaar, M., Eikland, K., Notebaert, P., et al. (2004). lpsolve: Open source (mixed-integer) linear programming system. Eindhoven U. of Technology, 63.

da Silveira Rizzo, E.M. (2009). Processo de fabricação de ferro-gusa em alto-forno. ABM.

Geerdes, M., Chaigneau, R., and Kurunov, I. (2015). Modern Blast Furnace Ironmaking: An Introduction (2015). Ios Press.

Griva, I., Nash, S.G., and Sofer, A. (2009). Linear and nonlinear optimization, volume 108. Siam.

James, G., Witten, D., Hastie, T., and Tibshirani, R. (2013). An introduction to statistical learning, volume 112. Springer.

Nocedal, J. and Wright, S. (2006). Numerical optimization. Springer Science \& Business Media.

Wu, M., Zhang, K., An, J., She, J., and Liu, K.Z. (2018). An energy efficient decision-making strategy of burden distribution for blast furnace. Control Engineering Practice, 78, 186-195.

Wu, S., Han, H., and Liu, X. (2010). Mathematical model for blast furnace burden optimization based on the hightemperature reactivity. ISIJ international, 50(7), 987993.

Yang, Y., Yin, Y., Wunsch, D., Zhang, S., Chen, X., Li, X., Cheng, S., Wu, M., and Liu, K.Z. (2017). Development of blast furnace burden distribution process modeling and control. Isij International, ISIJINT-2017.

Zhang, Y., Zhou, P., and Cui, G. (2018). Multi-model based pso method for burden distribution matrix optimization with expected burden distribution output behaviors. IEEE/CAA Journal of Automatica Sinica. 